\title{
Commentary: Warm versus cold cardioplegia: The devil is in the details
}

\author{
Victor A. Ferraris, MD, PhD
}

In this issue of the Journal, Ming Kot and colleagues ${ }^{1}$ present a meta-analysis of patients undergoing cardiac operations with either warm or cold cardioplegic arrest. The authors performed a meta-analysis of publications retrieved from all of the usual large publication databases (eg, Embase, Cochrane Library, PubMed) and identified studies that compared warm and cold cardioplegic arrest during cardiac operations. The authors concluded that there were no differences in safety outcomes between these 2 methods of temperature management of cardioplegia solutions. In this comparison of cold versus warm cardioplegia delivery, the authors simplified the multiple differences between cardioplegic solutions by focusing only on temperature and not on the multiple combinations of ingredients and delivery methods used in cardiac operations.

Cardioplegic solutions have some things in common, including high potassium concentrations to induce arrest, as well as a balanced electrolyte composition to mimic whole blood electrolyte levels. However, variations in the types and compositions of cardioplegic solutions, as well as the abundance of delivery methods, are almost too numerous to count. In sorting out differences in efficacy and safety of cardioplegia delivery for ischemic arrest, the "devil is in the details." It seems exceedingly simplistic to focus only on "warm versus cold" cardioplegia solutions to induce cardiac arrest.

\footnotetext{
From the Division of Cardiothoracic Surgery, University of Kentucky and Lexington VA Medical Center, Lexington, Ky.

Disclosures: The author reported no conflicts of interest.

The Journal policy requires editors and reviewers to disclose conflicts of interest and to decline handling or reviewing manuscripts for which they may have a conflict of interest. The editors and reviewers of this article have no conflicts of interest.

Received for publication April 6, 2021; revisions received April 6, 2021; accepted for publication April 13, 2021; available ahead of print May 5, 2021.

Address for reprints: Victor A. Ferraris, MD, PhD, Department of Cardiothoracic Surgery, University of Kentucky, A301, Kentucky Clinic, 740 South Limestone, Lexington, KY 40536-0284 (E-mail: ferraris@uky.edu).

JTCVS Open 2021;6:191-2

2666-2736

Copyright (C) 2021 The Author(s). Published by Elsevier Inc. on behalf of The American Association for Thoracic Surgery. This is an open access article under the CC BY-NC-ND license (http://creativecommons.org/licenses/by-nc-nd/4.0/).

https://doi.org/10.1016/j.xjon.2021.04.006
}

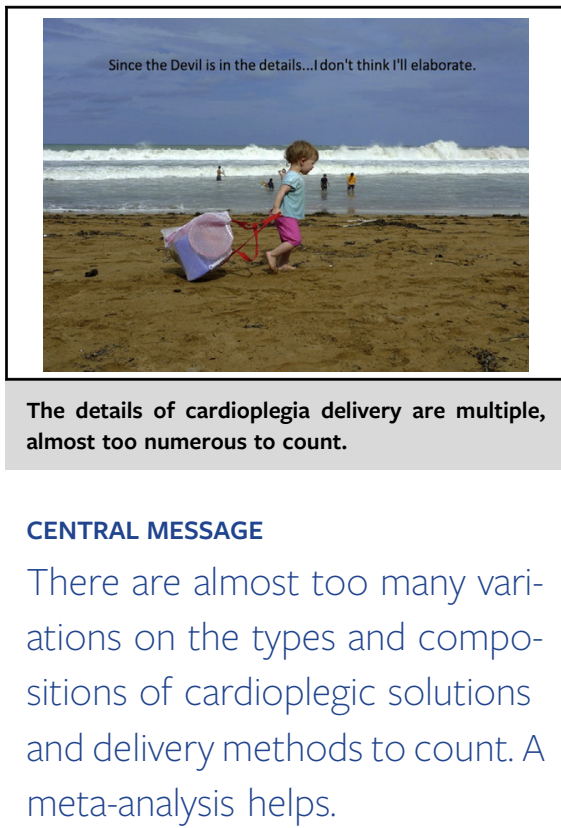

The investigation of cardioplegic solutions and their benefits is not a new area of study. ${ }^{2}$ Almost 30 years ago, Buckberg wrote an editorial suggesting that cardiac surgeons "should refrain from an inflexible adherence to a single approach for cardioplegia delivery based on an adversarial position that results in a self-imposed and counterproductive dilemma." 3 Apparently his editorial did not hinder the debate about types of cardioplegia and delivery methods. Since that time, there have been more than 50 comparisons written about the value of cold versus warm cardioplegia, crystalloid versus blood cardioplegia, antegrade versus retrograde delivery, and the value of all sorts of cardioplegic solution additives. There is a robust literature investigating the value and safety of various cardioplegia solutions and administration techniques. In this issue of the Journal, Ming Kot and colleagues sum up a portion of this literature with their meta-analysis. In attempting to sort out differences in the surgical results associated with cardioplegic arrest during cardiac operations, it would be helpful (almost mandatory) to categorize patients by the exact method of cardioplegia delivery used and by the exact compositions of the cardioplegic solutions administered. To assume that cardioplegic arrest depends only on whether a cold solution or a warm solution was used is simplistic at best and possibly misleading at worst.

There are some uncertainties and some recognized limitations in the myriad of cardioplegia compositions and in the multiple delivery systems of cardioplegia: 
1. One method of cardioplegia delivery that requires scrutiny involves the delivery of cardioplegic solution through saphenous vein grafts. The available data suggest that certain cardioplegic solutions delivered through vein grafts may cause marked vasomotor effects including spasm, and ultimate compromise of saphenous vein grafts. ${ }^{4}$

2. There may be a signal that warm cardioplegia is associated with adverse neurologic outcomes in certain patients with preexisting cerebrovascular disease.

3. Some questions about the choice of cardioplegia additives and delivery in children remain unanswered. Likewise, there are questions about the choice and delivery of cardioplegia in patients with extended cross-clamp times. $^{5}$

4. There are some uncertainties about the impact of certain cardioplegia additives on myocardial microRNA and the regulation of myocardial gene expression. ${ }^{6}$

There have been multiple summaries and evidence-based synopses of the types and delivery methods of cardioplegia solutions. One well-written and rigorous evidence summary was constructed by Jacob and colleagues, ${ }^{7}$ who used a structured protocol to obtain the best evidence about a comparison of blood versus crystalloid cardioplegia solutions. A robust literature search reviewed factors that affect cardioplegia success, including warm or cold blood cardioplegia, antegrade and retrograde administration, systemic hypothermia or normothermia, topical heart cooling, high and low potassium solutions, "hot shots," warm induction, volume of cardioplegia, patient factors, and bypass times. They screened more that 500 publications and ultimately used 22 manuscripts to formulate the "best evidence for safe and effective cardioplegia delivery." Important findings from this summary suggest that (1) there was a lower incidence of low output syndrome and CK-MB release with blood cardioplegia, and (2) there was a statistically significant decreased cardiac enzyme release associated with blood cardioplegia.
Despite these apparent reported benefits associated with blood cardioplegia, a survey of cardioplegia use in Britain suggested that $56 \%$ of surgeons use cold blood cardioplegia, $14 \%$ use warm blood cardioplegia, $14 \%$ use crystalloid cardioplegia, $21 \%$ use retrograde infusion, and $16 \%$ do not use any cardioplegia.

Summarizing findings is a difficult job, given the myriad reports favoring one type or delivery method of cardioplegia. The current manuscript by Ming Kot and colleagues provides an updated modern version of preferred cardioplegia delivery. It is quite likely that "one size does not fit all," but there is a recurring signal that blood cardioplegia has some benefits that favor its use over crystalloid cardioplegia. As the complexity of cardiac procedures increases, a "gray area" remains that requires more investigation with regard to the nuances of cardioplegia types and delivery systems. So, despite the known benefits of blood cardioplegia, there are still some unanswered questions that need to be addressed.

\section{References}

1. Ming Kot TK, Kai Chan JS, Froghi S, Hei Lau DH, Morgan K, Magni F, et al. Warm versus cold cardioplegia in cardiac surgery: a meta-analysis with trial sequential analysis. J Thorac Cardiovas Surg Open. 2021;162:161-90.

2. Guyton RA, Gott JP, Brown WM, Craver JM. Cold and warm myocardial protection techniques. Adv Card Surg. 1996;7:1-29.

3. Buckberg GD. Warm versus cold blood cardioplegia: a self-imposed and counterproductive dilemma. Ann Thorac Surg. 1993;56:1007-10.

4. Chiavarelli M, Fabi F, Stati T, Chiavarelli R, del Basso P. Effects of cardioplegic solutions and their components on human saphenous vein contractility. Ann Thorac Surg. 1992;53:455-9.

5. Liakopoulos OJ, Kuhn EW, Choi YH, Chang W, Wittwer T, Madershahian N, et al. Myocardial protection in cardiac surgery patients requiring prolonged aortic crossclamp times: a single-center evaluation of clinical outcomes comparing two blood cardioplegic strategies. J Cardiovasc Surg (Torino). 2010;51:895-905.

6. Kiss A, Heber S, Kramer AM, Hackl M, Skalicky S, Hallström S, et al. MicroRNA expression profile changes after cardiopulmonary bypass and ischemia/ reperfusion-injury in a porcine model of cardioplegic arrest. Diagnostics (Basel). 2020; $10: 240$.

7. Jacob S, Kallikourdis A, Sellke F, Dunning J. Is blood cardioplegia superior to crystalloid cardioplegia? Interact Cardiovasc Thorac Surg. 2008;7:491-8. 